\title{
Mass, Confinement and CP Invariance in the Seiberg-Witten Model
}

\author{
Massimo Di Pierro ${ }^{1}$ and Kenichi Konishi ${ }^{2}$ \\ 1 Dipartimento di Fisica - Università di Pisa \\ Istituto Nazionale di Fisica Nucleare - sez. di Pisa \\ Piazza Torricelli, 2 - 56100 Pisa (Italy) \\ E-mail:df144023@ipifidpt.difi.unipi.it \\ 2 Dipartimento di Fisica - Università di Genova \\ Istituto Nazionale di Fisica Nucleare - sez. di Genova \\ Via Dodecaneso, 33 - 16146 Genova (Italy) \\ E-mail: KONISHI@GE.INFN.IT
}

\begin{abstract}
Several physics aspects of the Seiberg-Witten solution of $N=2$ supersymmetric Yang-Mills theory with $S U(2)$ gauge group, supplemented with a small mass term for the "matter" fields which leads to an $N=1$ theory with confinement, are discussed. The light spectrum of the theory is understood on the basis of current algebra relations and the $\mathrm{CP}$ invariance of the massless and massive theories are studied. We find that in the massive (confining) theory the low energy physics has an exact $\mathrm{CP}$ symmetry, while in a generic vacuum in the massless theory $\mathrm{CP}$ invariance is broken spontaneously.
\end{abstract}

GEF-Th-6/1996

May 1996 
In a celebrated work [1] Seiberg and Witten exploited the (generalized) electromagnetic duality, $N=2$ supersymmetry and holomorphic property of effective actions, to solve exactly a strongly interacting non Abelian theory in four dimensions, i.e., to compute the vacuum degeneracies, and in each vacuum, to determine the exact spectrum and interactions among light particles.

An especially interesting observation of Ref [1], made in the pure $N=2$ supersymmetric YangMills theory with $S U(2)$ gauge group, is that upon turning on the mass term $\int d^{2} \theta \operatorname{Tr} m \Phi^{2}, m \ll \Lambda$, the light magnetic monopole field condenses, providing thus the first explicit realization of the confinement mechanism envisaged by 't Hooft. [2] In this note we wish to make some further observations on this model.

There are in fact several related questions which to our knowledge have not yet been discussed fully. Why do the vacua in the massive (confining) theory correspond precisely to those points of the quantum moduli space (QMS) of the $N=2$ theory where the magnetic monopole becomes massless? Usually, one expects that a dynamically generated mass in a non Abelian theory with scale $\Lambda$ is of the order of $\Lambda$, while in this model the mass gap is of the order of $m^{1 / 2} \Lambda^{1 / 2} / \log ^{1 / 2}(\Lambda / m)$ (see below). Why is that so? Is the CP invariance spontaneously broken at low energies? Does the oblique confinement 22 take place? Do dyons condense? What is the relation between the Seiberg-Witten effective action and the more speculative (but supposedly exact) effective superpotential constructed for the $N=1$ theory with the "integrating in" procedure? [3] Finally, has all this got anything to do with what happens in an $N=0$ theory of interest such as QCD?

A first general remark is that one is here dealing with a system with large vacuum degeneracy, intact after full quantum corrections are taken into account. This vacuum degeneracy is (almost) eliminated by the mass perturbation, $\int d^{2} \theta \operatorname{Tr} m \Phi^{2}$, leaving only a double degeneracy corresponding to the order parameter $u=\left\langle\operatorname{Tr} \phi^{2}\right\rangle= \pm \Lambda^{2}$.

As is usual in the standard degenerate perturbation theory in quantum mechanics, to lowest order the only effect of the perturbation is to fix the vacuum to the "right", but unperturbed, one, with $N=2$ (hence $S U_{R}(2)$ ) symmetric properties. This means that the $S U_{R}(2)$ current of the original theory,

$$
J_{\mu}^{a}=\operatorname{Tr} \bar{\Psi} \bar{\sigma}_{\mu} \tau^{a} \Psi
$$

where $\Psi=\left(\begin{array}{c}\lambda \\ \psi\end{array}\right)$, is well approximated in the low energy effective theory by the Noether currents (of the low energy theory)円

$$
J_{\mu}^{a}=\bar{\Psi}_{D} \bar{\sigma}_{\mu} \tau^{a} \Psi_{D}+i\left(D_{\mu} S^{\dagger}\right) \tau^{a} S-i S^{\dagger} \tau^{a} D_{\mu} S
$$

where $\Psi_{D}=\left(\begin{array}{c}\lambda_{D} \\ \psi_{D}\end{array}\right)$ is the dual of the (color diagonal part of) $\Psi$, and $S \equiv\left(\begin{array}{c}M \\ \tilde{M}^{\dagger}\end{array}\right)$. In arriving at Eq.(2) we identified the high energy and low energy $S U_{R}(2)$ groups such that the doublet $\left(\begin{array}{l}\lambda_{D} \\ \psi_{D}\end{array}\right)$

\footnotetext{
${ }^{1}$ We follow the notation of Ref[1].

${ }^{2}$ The effective low energy action is $\mathbb{4} \frac{1}{4 \pi} \operatorname{Im}\left[\int d^{4} \theta\left(\partial F\left(A_{D}\right) / \partial A_{D}\right) \bar{A}_{D}+\int d^{2} \theta\left(\partial^{2} F\left(A_{D}\right) / \partial A_{D}^{2}\right) W_{D} W_{D} / 2\right]$, with the addition of the standard terms for the magnetic monopole sector, $M^{\dagger} e^{V_{D}} M+\left.\tilde{M}^{\dagger} e^{-V_{D}} \tilde{M}\right|_{D}+\left.\sqrt{2} A_{D} \tilde{M} M\right|_{F}+h . c$. The mass term is geiven by $\int d^{2} \theta m \Phi^{2}+h . c .=\int d^{2} \theta m U\left(A_{D}\right)+h . c$.
} 
transforms as $\left(\begin{array}{l}\lambda \\ \psi\end{array}\right)$.

Let us consider the current-current correlation function

$$
i \int d^{4} x e^{-i q x}\left\langle 0\left|T\left\{J_{\mu}^{3}(x) J_{\nu}^{3}(0)\right\}\right| 0\right\rangle=\left(q_{\mu} q_{\nu}-q^{2} g_{\mu \nu}\right) \Pi\left(q^{2}\right),
$$

and an associated $R$-like ratio,

$$
R=\operatorname{Disc}_{q^{2}} \Pi\left(q^{2}\right),
$$

appropriately normalized (by introducing hypothetic "leptons"). At high energies $R$ just counts the number of $\lambda$ and $\psi$ "quarks" (asymptotic freedom), apart from calculable logarithmic corrections as well as an infinite number of not-so-easily-calculable power corrections (involving gluonic and higher condensates):

$$
R_{q^{2} \gg \Lambda^{2}} \simeq 6+O\left(\alpha\left(q^{2}\right), q^{4} / \Lambda^{4}\right) .
$$

At low energies, the same quantity is simply given by the weakly interacting dual fields and magnetic molopoles,

$$
R_{m \Lambda \ll q^{2} \ll \Lambda^{2}} \simeq 3,
$$

where two out of three comes from the contributions from $\lambda_{D}$ and $\psi_{D}$ (one each) and one from $M$ and $\tilde{M}$ (one half each). This amounts to an exact resummation of an infinite number of power and logarithmic corrections.

To next order the effect of the explicit $S U_{R}(2)$ breaking,

$$
\begin{gathered}
\partial^{\mu} J_{\mu}^{-}=\partial^{\mu} \operatorname{Tr} \bar{\psi} \bar{\sigma}_{\mu} \lambda=i m \operatorname{Tr} \lambda \psi ; \\
\partial^{\mu} J_{\mu}^{3}=\frac{1}{2} \partial^{\mu} \operatorname{Tr}\left(\bar{\lambda} \bar{\sigma}_{\mu} \lambda-\bar{\psi} \bar{\sigma}_{\mu} \psi\right)=\frac{i}{2}\left(\operatorname{Tr} m \psi^{2}-\text { h.c. }\right)
\end{gathered}
$$

must be taken into account. In particular, the anomaly of Ref 4

$$
m u=m\left\langle\operatorname{Tr} \phi^{2}\right\rangle=2 \frac{g^{2}}{32 \pi}\langle\operatorname{Tr} \lambda(x) \lambda(x)\rangle,
$$

hence

$$
\left(g^{2} / 32 \pi\right)\langle\operatorname{Tr} \lambda(x) \lambda(x)\rangle= \pm m \Lambda^{2} / 2
$$

implies that the $S U_{R}(2)$ symmetry is spontaneously broken also (which may be called induced breaking). . As $\lambda^{2}$ condensate is a triplet it breaks $S U_{R}(2)$ symmetry completely, implying three light pseudo Nambu-Goldstone particles. Also, the supersymmetric Ward-Takahashi like identity

$$
\left\langle\operatorname{Tr} \psi^{2}\right\rangle=2 m^{*}\left\langle\operatorname{Tr} \phi^{*} \phi\right\rangle
$$

\footnotetext{
${ }^{3}$ Although we are discussing here the first order mass corrections in a degenerate perturbation theory, many crucial relations are exact due to the nonrenormalization theorem (the form of the superpotential, etc). Most results below, in particular the exact CP invariance of the low energy theory, should survive higher order corrections which affect only the D type terms.

${ }^{4}$ This is similar to what happens to the isospin invariance in QCD: although the main dynamical effect (quark condensation) is isospin invariant, the addition of unequal up and down quark masses causes the respective condensates to take a little different values, thus breaking spontaneouly the isopspin, very slightly.
} 
and the fact that $\phi$ is in the adjoint representation hence probably $\left\langle\operatorname{Tr} \phi^{*} \phi\right\rangle \sim \operatorname{cost} \cdot\left\langle\operatorname{Tr} \phi^{2}\right\rangle \simeq \Lambda^{2}$, suggests that $\left\langle\operatorname{Tr} \psi^{2}\right\rangle \sim O\left(m \Lambda^{2}\right)$ also.

The associated "pion decay constants" can be easily read off from the expression of the low energy currents Eq.(2). Let us recall that upon turning on the mass term the vacuum is found to be fixed at $A_{D}=0, u=\Lambda^{2}$ as noted by Seiberg and Witten. [1] The superpotential $\sqrt{2} A_{D} \tilde{M} M+m U\left(A_{D}\right)$, minimized with respect to $A_{D}, \tilde{M}$ and $M$, indeed yields $A_{D}=0$ and the magnetic monopole condensation,

$$
\langle M\rangle=\langle\tilde{M}\rangle=\left(-m U^{\prime}(0) / \sqrt{2}\right)^{1 / 2} .
$$

Expanding the magnetic monopole fields around its vacuum expectation value

$$
M=\langle M\rangle+M^{\prime}, \quad \tilde{M}=\langle M\rangle+\tilde{M}^{\prime},
$$

one finds that

$$
F_{\pi}^{-} \sim F_{\pi}^{3} \sim\langle M\rangle=O\left(m^{1 / 2} \Lambda^{1 / 2}\right) .
$$

Also, it is easy to see that the three pseudo Nambu Goldstone bosons are (to lowest order) the real and imaginary parts of $M-\tilde{M}$ and the imaginary part of $\left\langle M^{\dagger}\right\rangle(M+\tilde{M})$, apart from normalization. (Actually, a linear combination of the real and imaginary parts of $M-\tilde{M}$ becomes the longitudinal part of the dual vector boson by the Higgs mechanism.)

As for the masses of the light particles, they can be studied most easily from the fermion bilinear terms arising from the Yukawa interaction terms upon shifting the magnetic monopole fields (Eq.(13)). It reads

$$
\begin{aligned}
L_{Y} & \left.=\sqrt{2}\left[-\left\langle M^{\dagger}\right\rangle \lambda_{D}\left(\psi_{M}-\psi_{\tilde{M}}\right)+\langle M\rangle \psi_{D}\left(\psi_{\tilde{M}}+\psi_{M}\right)\right]+\frac{m}{2} U^{\prime \prime}(0) \psi_{D} \psi_{D}\right]+ \\
& +\sqrt{2}\left[-M^{\prime \dagger} \lambda_{D} \psi_{M}+\tilde{M}^{\prime \dagger} \lambda_{D} \psi_{\tilde{M}}-\left(A_{D} \psi_{M} \psi_{\tilde{M}}+\psi_{D} \psi_{\tilde{M}} M^{\prime}+\psi_{D} \psi_{M} \tilde{M}^{\prime}\right)\right] \\
& +\frac{m}{2}\left(U^{\prime \prime}\left(A_{D}\right)-U^{\prime \prime}(0)\right) \psi_{D} \psi_{D}+\text { h.c. }
\end{aligned}
$$

A subtle point in reading off the masses from Eq.(15) is that since the fields $\lambda_{D}$ and $\psi_{D}$ do not have the canonical kinematic terms, they must be re-normalized by $\lambda_{D} \rightarrow g_{D} \lambda_{D} ; \psi_{D} \rightarrow g_{D} \psi_{D}$. (In the formula (2) for the low energy currents such a rescaling has already been done.) In doing so the argument of the dual coupling constant $g_{D}$ should be taken as $(m / \Lambda)^{1 / 2}$ and not $a_{D}=0$, since in the massive theory there is an infrared cutoff. (Such a replacement should automatically take place if the perturbation in $m$ is pushed to higher order. An analogous phenomenon is known in the old chiral perturbation theory due to the small pion mass.) This explains the $\log (\Lambda / m)$ dependence of the masses below.

From Eq.(15) one sees that the fields $\lambda_{D}$ and $\psi_{2} \equiv\left(\psi_{M}-\psi_{\tilde{M}}\right) / \sqrt{2}$ form a Dirac type massive fermion with mass

$$
m_{1}=\left|2 g_{D}\left\langle M^{\dagger}\right\rangle\right|=2\left|g_{D} \cdot(\sqrt{2} i m \Lambda)^{1 / 2}\right|=\frac{4 \cdot 2^{3 / 4} \pi|m \Lambda|^{1 / 2}}{\log ^{1 / 2}|\Lambda / m|},
$$

while in the subspace of two Weyl fermions $\psi_{D}$ and $\psi_{1} \equiv\left(\psi_{M}+\psi_{\tilde{M}}\right) / \sqrt{2}$ the mass matrix reads

$$
\left(\begin{array}{cc}
m g_{D}^{2} U^{\prime \prime}(0) / 2 & g_{D}\langle M\rangle \\
g_{D}\langle M\rangle & 0
\end{array}\right),
$$


where $U^{\prime \prime}(0)=-1 / 2$. The phases of these matrix elements can be chosen all real and positive by an appropriate phase rotation of the $\psi_{D}$ and $\psi_{1}$ fields (more about these phases below). A real symmetric matrix can be diagonalized by a real orthogonal matrix, leading to mass eigenvalues

$$
m_{2,3} \simeq \gamma|m \Lambda|^{1 / 2} \pm \frac{1}{2} \delta|m|
$$

where

$$
\delta=\frac{g_{D}^{2}}{4} ; \quad \gamma=2^{1 / 4} g_{D} ; \quad g_{D}=\frac{2 \sqrt{2} \pi}{\log ^{1 / 2}|\Lambda / m|}
$$

The result, Eq.(16), Eq.(18), that all the light particles have mass, $m_{\pi} \sim O\left(m^{1 / 2} \Lambda^{1 / 2} / \log ^{1 / 2}(\Lambda / m)\right)$, as well as the result for $F_{\pi}$, Eq.(14), can be understood in the light of the Dashen's formula [ [ [

$$
\left|F_{\pi}\right|^{2} m_{\pi}^{2} \simeq m\left\langle\operatorname{Tr} \psi^{2}\right\rangle+\text { h.c. }
$$

which is obtained by saturating the current-current correlation functions such as Eq.(3) (at $q^{2} \leq$ $m \Lambda)$ by lowest lying particles. Eq. (20) is indeed satisfied if on the right hand side $\left\langle\operatorname{Tr} \psi^{2}\right\rangle \sim$ $m \Lambda^{2} / \log (\Lambda / m)$ which is quite reasonable in view of Eq.(11). 5 Also, the (order-of-magnitude) equality between $F_{\pi}^{-}$and $F_{\pi}^{3}$ and between $m_{\pi}^{-}$and $m_{\pi}^{0}$ is what is expected: the same operator $m \operatorname{Tr} \psi^{2}+$ h.c. appears on the right hand side of the Dashen's formula for the correlation functions, $T\left\{J_{\mu}^{+} J_{\nu}^{-}\right\}$and $T\left\{J_{\mu}^{3} J_{\nu}^{3}\right\}$.

The $N=1$ theory (with $m \ll \Lambda$ ) thus contains in its spectrum three light pseudo NambuGoldstone bosons whose masses vanish in the limit $m \rightarrow 0$ as $\sim m^{1 / 2}$, and their $(N=1)$ superpartners degenerate in mass. Since low energy chiral superfields $A_{D}$ and $W_{D}$ together contain only two scalars, we know a priori that there must be some massless scalars in the low energy effective theory other than these particles - the situation is saved by the light monopoles (which plays here the role of pseudo Nambu-Goldstone bosons)! In other words, the two $N=1$ vacua could not be anywhere else in the QMS of $N=2$ theory, except at the two singular points $u= \pm \Lambda^{2}$. Other points e.g., with $|u|=\left|\Lambda^{2}\right|$, correspond to theories with only two massless scalars (see Fig. 1 below) hence are not near any of the $N=1$ vacua.

Exactly the same physics arises if one starts with an effective action with the $( \pm 1, \pm 1)$ "dyons", $\tilde{N}, N$, coupled to $A_{D}+A$ fields: one finds that the introduction of a small mass term fixes the vacuum to be at $A_{D}+A=0\left(u=e^{+i \pi} \Lambda^{2}, \theta_{\text {eff }}=-2 \pi\right)$. Because of the Witten's effect $(q$ is the observed electric charge, $n_{e}, n_{m}$ are the integer quantum numbers labelling the particles)

$$
q=n_{e}+\left(\theta_{e f f} / 2 \pi\right) n_{m}
$$

the $(1,1)$ particle (which would be massive at $u=+\Lambda^{2}$ but massless at $u=e^{+i \pi} \Lambda^{2}$ ) actually becomes a pure magnetic monopole there and condenses, leading to confinement of the color electric charges. After a redefinition of the low energy fields by $A_{D}+A \rightarrow A_{D}$ (which is an allowed $S L(2, Z)$ transformation) and a relabelling of the quantum number $n_{e} \rightarrow n_{e}-n_{m}$, the theory in this vacuum becomes even formally identical to the case $u=\Lambda^{2}$ studied above. Thus the "dyon condensation"

\footnotetext{
5 The generalized Dashen's formula by Veneziano, [6] with $\sim m\left\langle\operatorname{Tr} \phi^{2}\right\rangle$ on the right hand side, here neither confirms nor contradicts these results. Due to the fact that the scalar $\phi$ is neutral with respect to $S U_{R}(2)$, it simply states that certain operator has no amplitudes to produce these pseudo Goldstone bosons from the vacuum.
} 
does not occur in this model, contrary to such a statement sometimes made in the literature. We may therefore limit ourselves here and below to the $u=\Lambda^{2}$ vacuum without losing generality.

In view of the light spectrum found here let us compare the massive Seiberg-Witten effective low energy theory to a more naïve guess for the structure of the effective action, constructed by introducing just two chiral superfields $U=\operatorname{Tr} \Phi^{2}$ and $S \equiv \frac{g^{2}}{32 \pi} \operatorname{Tr} W W$ and by using the so-called "integrating-in" procedure, which leads to the superpotential[3]

$$
S \log \frac{U^{2}}{\Lambda^{4}}+m U \text {. }
$$

Eq.(22) is such that the anomalous transformation of the action, $4 \alpha\left(g^{2} / 32 \pi\right) F_{\mu \nu} \tilde{F}^{\mu \nu}$ (under $\Phi \rightarrow$ $\left.e^{i \alpha} \Phi\right)$, is formally reproduced. Such an "effective action" yields upon minimization of the scalar potential, also the correct results, $u=\left\langle\operatorname{Tr} \Phi^{2}\right\rangle= \pm \Lambda^{2}$ and the anomaly Eq.(9). Nonetheless, it is an incorrect low energy action in this theory, as it does not contain light particles with mass $\sim m^{1 / 2} \Lambda^{1 / 2}$. The reason for such a failure seems to lie in the fact that the global $S U_{R}(2)$ symmetry (and its small induced breaking) in the limit of $m / \Lambda \rightarrow 0$ has not been taken into account properly.

It is of particular interest to study the way $\mathrm{CP}$ symmetry is realized in the massless $(N=2)$ and in the massive $(N=1)$ theories, and the relation thereof. Although one expects no dependence on the bare $\theta$ parameter even in the presence of the $\Phi$ mass term since in the original theory there is a massless charged fermion $(\lambda)$, ${ }^{6} \mathrm{CP}$ symmetry is realized at low energies in the Coulomb phase (massless case) and in the confining phase (massive case) in different ways. In the massless theory, the $\theta$ indepence is assured by the property of the exact Seiberg-Witten solution,

$$
a=\frac{\sqrt{2} \Lambda}{\pi} \int_{-1}^{1} d x \frac{\sqrt{x-u / \Lambda^{2}}}{\sqrt{x^{2}-1}} ; \quad a_{D}=\frac{\sqrt{2} \Lambda}{\pi} \int_{1}^{u / \Lambda^{2}} d x \frac{\sqrt{x-u / \Lambda^{2}}}{\sqrt{x^{2}-1}},
$$

in the following sense. A shift of $\theta$ by $\Delta \theta$ causes the change in $\Lambda$ as

$$
\Lambda \rightarrow e^{i \Delta \theta / 4} \Lambda \text {. }
$$

$\Lambda$ depends on $\theta$ this way since it depends on the bare coupling constant and bare $\theta$ parameter through the complex "coupling constant" $\tau=\theta / 2 \pi+4 \pi i / g^{2}$. [7] But if we now move to a different vacuum by

$$
u \rightarrow e^{i \Delta \theta / 2} u
$$

the net change is

$$
a \rightarrow e^{i \Delta \theta / 4} a ; \quad a_{D} \rightarrow e^{i \Delta \theta / 4} a_{D}:
$$

a common phase rotation of $a$ and $a_{D}$.

Thus all physical properties of an appropriately shifted vacuum $u$ with a new value of $\theta$ are the same as in the original theory. In particular, the full spectrum [1],

$$
M=\sqrt{2}\left|n_{m} a_{D}+n_{e} a\right| .
$$

as well as the low energy coupling constant and vacuum parameter are seen to be unchanged, since

$$
\tau_{\text {eff }}=\frac{\theta_{\text {eff }}}{2 \pi}+\frac{4 \pi i}{g_{\text {eff }}^{2}}=\frac{d a_{D}}{d a} .
$$

\footnotetext{
${ }^{6}$ We thank S. Hsu for pointing out an error in this regards which appeared in the original manuscript.
} 
In other words, the ensemble of theories represented by the points of QMS, taken together, is invariant under $\theta \rightarrow \theta+\Delta \theta$.

(The above argument may be inverted: the invariance of the theory under Eq.(24) and Eq.(25) can be interpreted as an indication that the anomalous chiral $U(1)$ transformation property of the theory is indeed correctly incorporated in the low energy action.)

On the other hand, at fixed generic $u$, physics depends on $\theta$ (as well as on the bare coupling constant $g$ ) non trivially: this is so because the CP invariance is spontaneously broken by $u=$ $\left\langle\operatorname{Tr} \Phi^{2}\right\rangle \neq 0$. Equivalently, at fixed given bare parameters there are theories with different value of $u$ and with inequivalent physics. For instance, the spectrum of some stable particles depends on $u$ (hence on the effective low energy coupling constant $g_{\text {eff }}$ and the theta parameter $\theta_{\text {eff }}$ ), if $u$ is smoothly changed along a semicircular path $u=e^{i \alpha} \Lambda^{2}, \alpha=0 \rightarrow \pi$, as is illustrated in Fig. 1.7 Note in particular the periodicity of the spectrum in $\theta_{\text {eff }}$ with periodicity $2 \pi$, in spite of a nontrivial spectral flow. Also, the theories at $u= \pm \Lambda^{2}$ are characterized by the fact that $\theta_{\text {eff }}=0$ (or $\pm 2 \pi$ which is the same as 0 because of the periodicity).

Consider now the massive theory. Upon turning on the mass term (even infinitesimal) the vacuum is fixed at $A_{D}=0, u=\Lambda^{2}$ in which the effectivelow energy vacuum parameter $\theta_{\text {eff }}$ takes the value zero. Nonetheless, since $u \neq 0$ (as in other vacua in the Coulomb phase) and also $\langle M\rangle \neq 0$, one might wonder whether CP invariance is broken spontaneously at low energies. In fact this does not occur.

To see that the low energy theory has indeed an exact $\mathrm{CP}$ invariance, let us go back to the Yukawa Lagrangian Eq.(15). By using Eq.(12) and the fact that $U^{\prime}(0)=-2 i \Lambda ; \Lambda=e^{i \theta / 4}|\Lambda|$, and $U^{\prime \prime}(0)=-1 / 2$ one sees that the only nontrivial phases appear in $m$ and $\langle M\rangle$,

$$
m=e^{i \alpha}|m|, \quad\langle M\rangle=e^{i \beta}|\langle M\rangle| ; \quad \alpha \equiv \arg m, \quad \beta \equiv \pi / 4+\arg m / 2+\theta / 8,
$$

as well as through the expansion of $U^{\prime \prime}\left(A_{D}\right)-U^{\prime \prime}(0)$ in powers of $A_{D} / \Lambda$. From the exact SeibergWitten formula for $a_{D}(u)$ (Eq.(23)) one finds by a change of the integration variable that

$$
\frac{a_{D}}{\Lambda}=\frac{i}{\pi} \sum_{n=0}^{\infty} \frac{(-)^{n} \Gamma^{2}(n+1 / 2)\left(u / \Lambda^{2}-1\right)^{n+1}}{2^{n+1}(n+1) ! n !}
$$

with an overall factor $i$ on the right hand side. By inverting the series one gets

$$
U\left(A_{D}\right)=\Lambda^{2}\left(1+f\left(A_{D} / i \Lambda\right)\right) ; \quad U^{\prime \prime}\left(A_{D}\right)=-f^{\prime \prime}\left(A_{D} / i \Lambda\right)
$$

where $f(x)=2 x+(1 / 4) x^{2}-(1 / 32) x^{3}+\ldots$ and $f^{\prime \prime}(x)=1 / 2-(3 / 16) x+\ldots$ are real functions of (possibly complex) variable $x$.

Now first make the phase rotations

$$
\psi_{D} \rightarrow e^{-i \alpha / 2} \psi_{D} ; \quad \psi_{M} \rightarrow e^{-i(\beta-\alpha / 2)} \psi_{M} ; \quad \lambda_{D} \rightarrow e^{i(2 \beta-\alpha / 2)} \lambda_{D} ; \quad M^{\prime} \rightarrow e^{i \beta} M^{\prime} .
$$

\footnotetext{
${ }^{7}$ It is important that such a path is taken outside the curve on which the ratio $a_{D} / a$ is real and on which the spectrum changes discontinuously, some stable particle becoming unstable, etc. According to Ref [8] it is a near ellipse included entirely in the unit circle $|u|=\left|\Lambda^{2}\right|$ except at the points $u= \pm \Lambda^{2}$.
} 
$\left(\psi_{\tilde{M}}\right.$ and $\tilde{M}^{\prime}$ transforms respectively as $\psi_{M}$ and $\left.M^{\prime}\right)$. These transformations eliminate phases from all masse terms as well as from the Yukawa terms involving $M^{\prime}$ 's. On the other hand the Yukawa term $A_{D} \psi_{M} \psi_{\tilde{M}}$ acquires a phase factor $\exp -2 i(\beta-\alpha / 2)=\exp (-i \pi / 2-i \theta / 4)$. The final rotation

$$
A_{D} \rightarrow e^{i \pi / 2+i \theta / 4} A_{D}
$$

however eliminates this phase from the Yukawa term and simultaneously transforms the argument of $f^{\prime \prime}(x)$ as $A_{D} / i \Lambda \rightarrow A_{D} /|\Lambda|$, making the expansion coefficients of $U^{\prime \prime}\left(A_{D}\right)-U^{\prime \prime}(0)$ in $A_{D}$ all real.

Thus the low energy effective theory is indeed independent of the bare $\theta$ parameter. More important, the spontaneous breakdown of CP invariance à la T.D. Lee[9] does not occur, all masses and Yukawa interaction coefficients being real. The low energy vacuum parameter $\theta_{\text {eff }}$ is zero. This completes the proof of CP invariance in the low energy theory. \& Since the low energy physics does not depend on the phase $\theta$, no oblique confinement speculated by t'Hooft (condensation of $(1,0)$ $(1,1)$ dyon pairs with opposite electric charges) [2] takes place in this model.

One might be tempted to conclude that, by using a similar argument as above, "spontaneous CP violation" does not occur a fortiori in the $m=0$ theory either, since $L_{Y}$ is much simpler in this case (no explicit mass term, no magnetic monopole condensation). This is not so. First of all, at a generic vacuum $u \neq \pm \Lambda^{2}$ there is nonzero $\theta_{e f f} F_{\mu \nu} \tilde{F}^{\mu \nu}$ term which breaks CP (this is also a spontaneous breaking since the nonzero $\theta_{\text {eff }}$ is due to the vacuum expectation value of $\operatorname{Re} d A_{D} / d A$ ). It is true that one can transform away $\theta_{\text {eff }}$ by an $S L(2, R)$ transformation of the scalar $A_{D} \rightarrow A_{D}+\left(\theta_{\text {eff }} / 2 \pi\right) A$; $A \rightarrow A$, which leaves the rest of the effective Lagrangian invariant. Such a transformation however introduces the Yukawa term of the form

$$
\sqrt{2}\left\{A_{D}+\left(\theta_{e f f} / 2 \pi\right) A\right\} \psi_{M} \psi_{\tilde{M}} .
$$

Since the condensates $\left\langle A_{D}\right\rangle$ and $\langle A\rangle$ are in general relatively complex, no phase rotation can eliminate the phases completely from the Lagrangian: CP invariance is broken spontaneously in this case. It is interesting that the above shift of the dual scalar $A_{D}$ transforms Witten's boundary effect Eq.(21) - the electric charge of the magnetic monopole, $\theta_{\text {eff }} / 2 \pi$, - into the standard (albeit mutually nonlocal) minimal couplings of $M$ with $A_{D \mu}$ and $A_{\mu}$, as can be seen from the $N=2$ supersymmetric completion of the Yukawa interaction, Eq.(34).

One thus reaches an amusing conclusion that the massless theory depends (in a given vacuum) on the $\theta$ parameter, while the massive theory is independent of it!

The low energy CP invariance and non-renormalization of the $\theta$ parameter in the massive case may be closely connected to the phenomenon of confinement. According to 't Hooft [2], the confinement is a sort of dual superconductivity, due to the condensation of (color) magnetic charges. Now if the dynamics of magnetic condensation is such that the magnetic monopoles must have rigorously zero electric charge to be able to condense, then it follows that by Witten's formula Eq.(21) the low energy $\theta$ parameter must be exactly zero. (For somewhat related ideas see Ref. [10].) This seems to be precisely what happens in the massive Seiberg-Witten model. However, since the independence

\footnotetext{
${ }^{8}$ As a further check, note that both sides of the Dashen's formula Eq.(2q) is indeed independent of the phase $\theta$ : $F_{\pi}$ enters as the absolute value squared, $m_{\pi}$ is real, and on the right hand side, the condensate $m\left\langle\psi^{2}\right\rangle$ is real as can be seen from the supersymmetric Ward-Takahashi identity, Eq.(11).
} 
of the low energy theory on the bare $\theta$ parameter is due to the presence of a massless fermion in the original model, an aspect probably not shared by the ordinary QCD, it is not clear whether the massive Seiberg -Witten model can be regarded as a good model of solution of the strong CP puzzle.

Let us conclude with a general comment. The bare $\theta$ parameter (which can be set to zero) is renormalized in the infrared by multi-instanton effects (or loops of dyons in the dual variables) differently in various vacua, i.e., to a nonzero value in a generic vacuum of the $N=2$ theory, to zero in the confining phase ( $N=1$ theory). Precisely these instanton effects are responsible for maintaining at any scale the duality relation $\tau_{D}=-1 / \tau, \tau=\theta_{\text {eff }} / 2 \pi+4 \pi i / g_{\text {eff }}^{2}$, which generalizes the Dirac's quantization condition 11] $g e=2 \pi n, \quad n=0,1,2, \ldots$. Note how an old puzzle related to the Dirac's quantization condition (how to maintain the quantization condition for $g$ and e which are both U(1) coupling constants hence which get renormalized smoothly in the same direction as the scale is slowly varied?) [12] is solved in the Seiberg-Witten model. The "electric" coupling constant $g_{\text {eff }}$ here is truly a non Abelian charge and gets renormalized in the opposite way compared to the $U_{D}(1)$ magnetic charge $g_{D}$. This consideration seems to strengthen the idea that magnetic monopoles and dyons can appear in Nature only as composite, solitonic particles in the context of a non Abelian gauge theory, spontaneously broken (or gauge-projected) to a group involving $U(1)$ subgroups.

\section{Acknowledgments}

One of the authors (K.K.) thanks LPTHE, Centre d'Orsay, Université de Paris-Sud where his study on these problems started, for a warm hospitality, the organizer and partecipants of the Kyoto workshop on Supersymmetry (March 96) for an occasion to present and discuss some of the subjects treated here, and his collegues A. Di Giacomo, T. Eguchi, Riccardo Guida, T. Kugo, G. Paffuti, P. Rossi, Hiroshi Suzuki, E. Tomboulis and Sung-Kil Yang for discussions.

\section{References}

[1] N. Seiberg and E. Witten, Nucl. Phys. B426 (1994) 19;

[2] G. 't Hooft, Nucl. Phys. B190[FS3] (1981) 455;

[3] N. Seiberg and K. Intrilligator, Nucl. Phys. B431 (1994) 551;

[4] K. Konishi, Phys. Lett. B135 (1984) 439;

[5] R. F. Dashen, Phys. Rev 183 (1969) 1245; ibid D3 (1971) 1879;

[6] G. Veneziano, Phys. Lett. 128B (1983) 199

[7] M.A. Shifman and A.I. Vainshtein, Nucl. Phys. B359 (1991) 571;

[8] U. Lindström and M, Rocek, Phys. Lett. 355B (1995) 492, A. Fayyazuddin, Mod. Phys. Lett. A10 (1995) 2703, P. Argyres, A. Faraggi and A. Shapere, hep-th/9505190; M. Matone, hepth/9506181, F. Ferrari and A. Bilal, hep-th/9602082;

[9] T.D. Lee, Physics Reports C 9 (1974) 143; 
[10] G. Schierholz, Nucl. Phys. B(Proc. Suppl.) 37A (1994) 203;

[11] P.A.M. Dirac, Proc. Roy. Soc. A133 (1931) 60;

[12] B. Zumino, Erice Lectures (1966), Ed. A. Zichichi; S. Coleman, Erice Lectures (1977), Ed. A. Zichichi.

\section{Figure Caption}

Fig.1 Mass spectrum of some stable particles with magnetic charge in the $N=2$ theory as $u$ varies as $u=e^{i \alpha} \Lambda^{2}, \alpha=0 \rightarrow \pi$. The numbers near each curve indicate $\left(n_{m}, n_{e}\right)$. The unit of mass is $4|\Lambda| / \pi$.

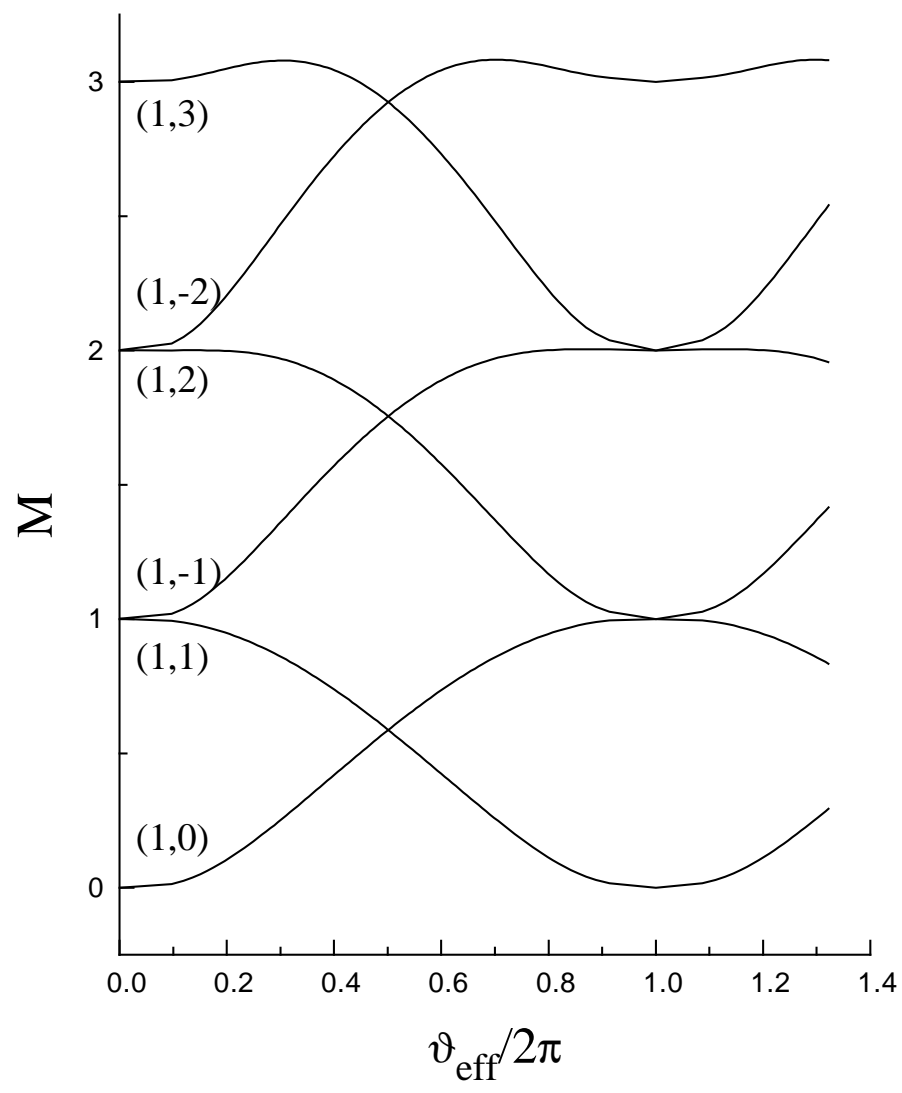

Figure 1: 